\title{
Peri-acetabular external fixation for hip disease: an anatomical study
}

\author{
Shaun Brown · Irwin Lasrado · Leo Donnan
}

Received: 11 August 2007/ Accepted: 3 October 2007/Published online: 9 November 2007

(C) Springer-Verlag 2007

\begin{abstract}
Hip distraction using cross joint articulated external fixation has been used by a number of orthopaedic centres for the treatment of osteoarthritis, chrondrolysis, and osteonecrosis, as an alternative to hip arthroplasty or arthrodesis in the adolescent and young adults. The hip, however, is problematic with respect to external fixation, as it lies deep, surrounded by powerful muscles and in intimate contact with major neurovascular structures and intrapelvic organs. The numbers of patients treated by this technique to date remains small and the technical detail and potential complications, with respect to the application of the external fixation, unclear. In this study we used anatomical information from CT scans combined with computer modelling of the hip and pelvis to identify safe screw positions in the periacetabular region. The surgical insertion of the pins was then performed on five cadavers and anatomical dissections undertaken to confirm the structures at risk. This study indicated that whilst there are a number of anatomic constraints, it is still possible to insert three pins with good divergence into the limited bony corridor of the peri-acetabular region recognising that the more posterior of the laterally inserted pins poses a potential risk to the sciatic nerve if not inclined away from the sciatic notch.
\end{abstract}

Keywords External fixation · Hip distraction · Anatomy

S. Brown · I. Lasrado · L. Donnan $(\bowtie)$

Department of Orthopaedic Surgery,

Royal Children's Hospital, Flemington Road, Parkville,

Melbourne, VIC 3052, Australia

e-mail: leo.donnan@rch.org.au

\section{Introduction}

External fixation around the pelvis has been used widely for the management of a number of orthopaedic conditions including traumatic disruption of the pelvic ring [1], arthrodeses of the hip [2] and as a stable skeletal base for spinal traction. More recently pelvic external fixation has been utilised to manage a variety of the hip pathologies that have proven difficult to treat by any other method. Arthrodiastasis (distraction) of the hip joint can be used in the management of osteoarthritis [3], chondrolysis [4] and osteonecrosis [5, 6, 7] of various aetiologies including Perthes disease, slipped upper femoral epiphyses, fractured neck of femur and corticosteroid treatment. Movement of the hip joint can be achieved by the addition of an appropriately orientated hinge within the fixator, thus incorporating principles of motion derived from Salter's work on articular cartilage [8]. The combination of distraction with motion provides, in theory, the ideal environment in which the hip (articular cartilage and bone) can recover from insult.

The most commonly used fixators for arthrodiastasis of the hip are monolateral devices that utilise supra-acetabular and femoral fixation with a fixed uni-planar hinge and a distracting mechanism [9]. The success of these devices has been reported by a number of authors, but in each case there is limited detail of the operative technique, no analysis of the potential complications of pin placement and little discussion of the underlying stability of fixation.

Supra-acetabular fixation transfixes the gluteus medius and mimimus muscles, puts the superior gluteal neurovascular bundle and sciatic nerve at risk whilst exposing intrapelvic structures to drill or pin penetration. The zone of bony fixation is narrow and the pins are biomechanically compromised being under significant cantilever loading due to limited divergence in cancellous bone and 
significant loading from the force of distraction and the weight of the leg below the hip joint.

A number of studies have previously investigated pelvic pin fixation both in the iliac crest and at the level of the anterior inferior iliac spine (AIIS) [10]. These studies have been in reference to pelvic fractures managed utilising static external fixation. Anatomical studies relating to percutaneous peri-acetabular fixation are limited to the exploration of the approach to the anterior inferior iliac spine $[11,12]$. The aim of this study was to define the anatomical risks associated with peri-acetabular external fixation and to explore the possibility of improving external fixation in this region.

\section{Materials and methods}

The initial part of this study focused on the bony anatomy of the peri-acetabular region of the pelvis. The bone-targeted CT scans of both adult and adolescent pelvises were evaluated in the coronal, sagittal and transverse planes to determine the best positioning of three pins into this region utilising the anterior inferior spine and the lateral supraacetabular region.

Pin positions selected were:

(a) Anterolateral: a pin inserted from the AIIS posteromedially to just above the greater sciatic notch.

(b) Lateral pre-capital: a lateral pin inserted anterior to the apex of the acetabulum above the anterolateral pin.

(c) Lateral post-capital: a lateral pin inserted posterior and inferior to the apex of the acetabulum below the anterolateral pin.

Utilising a three-dimensional virtual cadaver (Surgical Multimedia Services, Melbourne, Australia), based on cross-sectional MRI and CT scan data, the positioning of the pins in the pelvis was reproduced in order to delineate the extent of muscle penetration, proximity to extra pelvic neurovascular structures and as an exercise to familiarise investigators with the planes of pin insertion, technical difficulties and positioning of the image intensifier prior to performing the procedure on anatomical specimens (Figs. 1, 2).

Five embalmed cadavers that had not previously undergone any surgery to the pelvis were obtained from the Anatomical Department at the University of Melbourne. Each cadaver was placed on a radiolucent Perspex table in the supine anatomical position. The right hemi-pelvis was used for this study and 6-mm Steinman pins were introduced through small stab incisions without blunt dissection to minimise disturbance of the underlying neurovascular

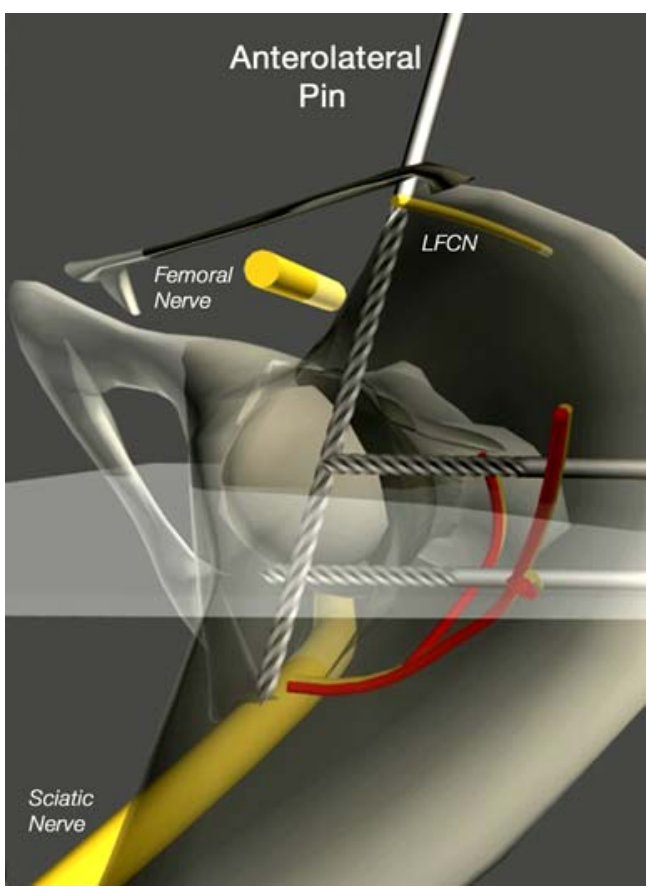

Fig. 1 Superior transverse view of pin positions in the peri-acetabular regions ( $L F C N$ lateral femoral cutaneous nerve)

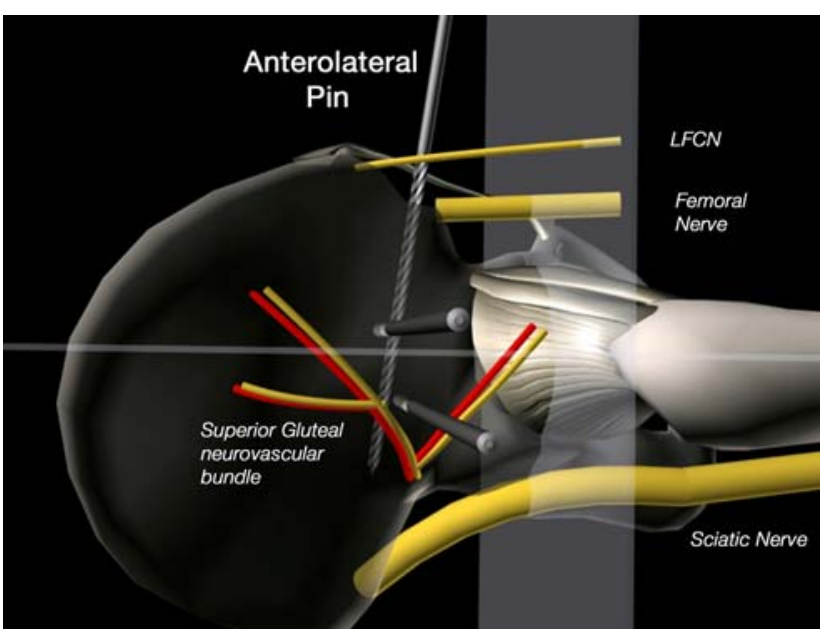

Fig. 2 Lateral view of pin positions in the peri-acetabular regions (LFCN lateral femoral cutaneous nerve)

structures. The length of the Steinman pins meant that they could be run deeply through the pelvis to determine what structures were directly at risk of damage by the inadvertent penetration of the bony pelvis.

All pins were introduced utilising fluoroscopy with the anterolateral (AIIS) pin acting as the reference for insertion of the lateral pins. The entry point of the insertion of the anterolateral pin was determined both on the anteroposterior and $30^{\circ}$ obturator oblique view with fluoroscopy as per the method described by Haidukewych 
et al. [11]. A "gothic arch" was visualised on the obturator oblique image and its centre was the guide for pin insertion whilst the $30^{\circ}$ internal iliac view was used to guide insertion above the greater sciatic notch. A true lateral image was obtained of the acetabulum and the apex was identified with a 1.6-mm K-wire. A targeting jig was fashioned from components from the Ilizarov system and attached to the anterolateral pin to assist in the insertion of the lateral pins, which were then driven deep into the pelvis.

Sequential dissections of the cadaveric specimens were then performed and measurements made from each pin to the important neurovascular structures utilising a digital Vernier calliper. All measurements were expressed as a mean distance from the pin to the object of interest at its closest point and the range of variation recorded. The relationship to pin penetration on the inner aspect of the pelvis and the intrapelvic structures was recorded. The pelvic specimens were then stripped of all soft tissue attachments, except for the hip joint capsule, and the positions of the pins recorded with reference to the acetabular margin and greater sciatic notch. The orientation between the pins was measured with a goniometer prior to transverse sectioning of the bony pelvis at each pin level. The tract of the pin was then photographed and measured to assess the degree of pin purchase in bone.

\section{Results}

Extrapelvic dissection

\section{Anterolateral pin}

The mean distance of the anterolateral (AIIS) pin to the lateral femoral cutaneous nerve (LFCN) was $6 \mathrm{~mm}$ (range $3-10 \mathrm{~mm}$ ) and to the femoral nerve (FN) was $35 \mathrm{~mm}$ (range $27-45 \mathrm{~mm}$ ). In three of the cadavers the LFCN was medial to the pins, in one the pin was found to be touching the medial border of the lateral (smaller) of the two branches of the LFCN and the nerve was not identified in one cadaver despite careful dissection and scrutiny. None of the nerves appeared to have been injured by any of the pins.

\section{Pre-capital pin}

All five pins were in the bone and were noted to have transfixed the muscle bellies of both the gluteus medius and minimus. The mean distance from the most inferior branch of the superior gluteal nerve (SGN) was $10 \mathrm{~mm}$ (range 0-25 mm) with four of the five pins passing superior to this branch. The mean distance from the deep inferior branch of the superior gluteal artery (SGA) was $11 \mathrm{~mm}$ (range 9-18 $\mathrm{mm}$ ); two pins were superior to the artery and three pins were inferior to it. The mean distance from the sciatic nerve (SN) was $46 \mathrm{~mm}$ (range 22-79 $\mathrm{mm}$ ) and from the pin to the bony edge of the greater sciatic notch was $37 \mathrm{~mm}$ (range 16-55 $\mathrm{mm}$ ).

\section{Post-capital pin}

One of the post-capital pins was noted to have poor bony purchase and on subsequent dissection was found to have entered the greater sciatic notch and obtained minimal bony contact. The other four pins were found in acceptable positioning in the bone.

All five pins were noted to have transfixed the muscle bellies of gluteus medius and minimus. The mean distance of the pins from the most inferior branch of the SGN was $9 \mathrm{~mm}$ below the nerve (range $1-19 \mathrm{~mm}$ ). The mean distance from the deep inferior branch of the SGA was $10 \mathrm{~mm}$ (range 4-19 $\mathrm{mm}$ ), four of the five pins were inferior to this branch and one was superior to it. The mean distances of the pins from the sciatic nerve was $30 \mathrm{~mm}$ (range 2-38 $\mathrm{mm}$ ) and from the bony edge of the greater sciatic notch $18 \mathrm{~mm}$ (range $0-33 \mathrm{~mm}$ ).

Intrapelvic dissection

\section{Anterolateral pin}

The depth of insertion of the anterolateral pin was deliberately exaggerated in order to determine if there was a potential to enter the sciatic notch. Under fluoroscopy it was felt that all pins were completely intraosseous and the only structure at risk was the sacroiliac joint. The deep dissection revealed that two pins had partially exited bone and entered the sciatic notch just medial to its apex. The superior gluteal neurovascular bundle and lumbosacral trunk transverse this region and are at potential risk.

\section{Pre-capital pin}

The majority of pins in this region enter the pelvis under cover of iliopsoas, which offers some protection to the intrapelvic structures. The external iliac artery and vein along with the obturator nerve are at particular risk in this region whilst the variability of the lumbosacral trunk and $\mathrm{S} 1$ nerve root can put these structures in close proximity (Table 1). 
Table 1 Relationship between lateral screws and major structures

\begin{tabular}{lll}
\hline Structure & $\begin{array}{l}\text { Pre-capital } \\
\text { mean distance } \\
(\mathrm{mm})\end{array}$ & $\begin{array}{l}\text { Post-capital } \\
\text { mean distance } \\
(\mathrm{mm})\end{array}$ \\
\hline Femoral nerve (intrapelvic) & $30(5-57)$ & $51(36-63)$ \\
Superior gluteal artery & $24(12-40)$ & $10(1-23)$ \\
Superior gluteal vein & $22(12-34)$ & $9(0-17)$ \\
Lumbosacral trunk & $23(2-29)$ & $7(0-22)$ \\
S1 nerve root & $32(4-50)$ & $16(3-32)$ \\
S2 nerve root & $46(26-51)$ & $25(14-40)$ \\
Obturator nerve & $11(0-29)$ & $17(9-18)$ \\
External iliac artery & $22(10-48)$ & $44(33-47)$ \\
External iliac vein & $11(0-32)$ & $30(18-44)$ \\
\hline
\end{tabular}

\section{Post-capital pin}

The more posterior location of this pin lessens the cover of the iliopsoas muscle and moves the entry point closer to the greater sciatic notch. The superior gluteal neurovascular bundle, the lumbosacral trunk and the S1 nerve root lie nearby with little overlying muscle protection (Table 1).

\section{Bony dissection}

\section{Anterolateral pin}

The average depth in the iliac bone was $102 \mathrm{~mm}$ (range 92-108 mm). Four pins had entered bone just lateral to the apex of the AIIS and two had partially exited the pelvis into the apex of the greater sciatic notch. The mean distance from the pin to the margin of the hip joint cavity was $15 \mathrm{~mm}$ (range 11-18 mm) (Fig. 3).

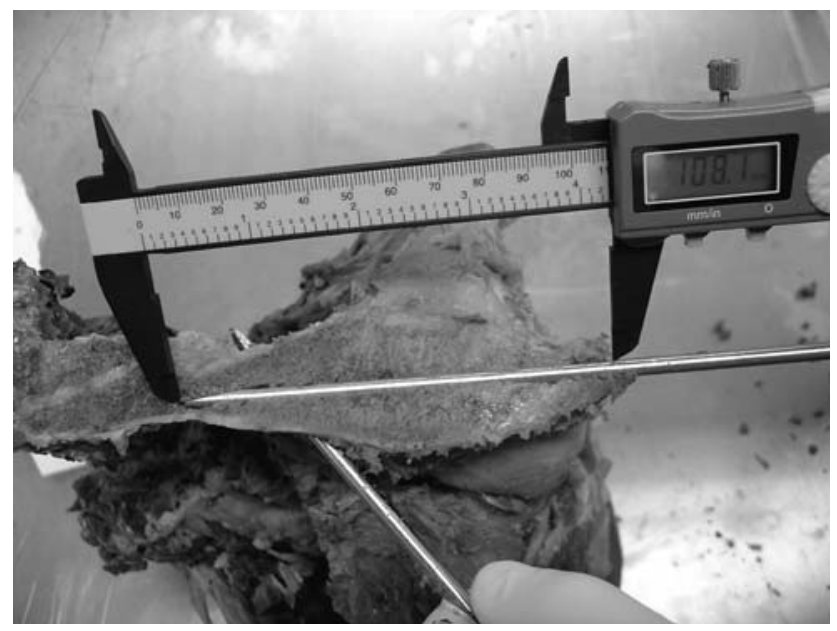

Fig. 3 Depth of bony penetration of the anterolateral pin

\section{Pre-capital pin}

The mean depth in bone was $32 \mathrm{~mm}$ (range $20-43 \mathrm{~mm}$ ) and the mean distance from the margin of the hip joint cavity was $22 \mathrm{~mm}$ (range 19-25 mm).

\section{Post-capital pin}

For the intraosseous pins the mean depth in bone was $26 \mathrm{~mm}$ (range $21-31 \mathrm{~mm}$ ) and the mean distance from the margin hip joint cavity was $9 \mathrm{~mm}$ (range $7-11 \mathrm{~mm}$ ).

\section{Pin orientation}

Mean angular separation (crossing angles) between the anterolateral and pre-capital pin was $56^{\circ}$ (range $40^{\circ}-78^{\circ}$ ) and between the anterolateral and post-capital pin it was $55^{\circ}\left(\right.$ range $\left.52^{\circ}-62^{\circ}\right)$.

\section{Discussion}

The lack of satisfactory treatment for a number of hip conditions promoted an interest in hip distraction as a conservative method in which the femoral head could be protected from collapse during healing after a vascular insult, distracted to rest compromised hyaline cartilage or articulated to overcome stiffness and promote healing. The effectiveness of such external fixation depends on the ability of the device to be safely and securely attached to the underlying skeleton in such a fashion that maximises patient comfort whilst the desired biological effect is achieved. Ilizarov [13] described hip distraction utilising a complex frame construct attached to the pelvis at multiple sites including the iliac crest and peri-acetabular region. The Ilizarov technique has been employed outside Russia and recently, Kucukkaya et al. [5] presented the results of a series of hip distractions with this device for the treatment of avascular necrosis of the femoral head in childhood. In the early 1980s Aldegheri [3] introduced the Orthofix monolateral hip distracter that utilised direct lateral periacetabular pin fixation with a hinge aligned to the transverse axis of the hip joint. Clinical series soon followed which promoted interest in this technique as a treatment for hip disease [14].

For the successful external fixation for hip distraction it is important to avoid damage to neurovascular structures and intrapelvic organs, obtain biomechanically stable and durable bony fixation whilst maximising patient comfort. The peri-acetabular region offers an ideal site containing the best quality and quantity of bone in the pelvis for pin 
insertion for achieving these aims whilst at the same time lying adjacent to the structure (hip joint) about which fixation is desired. Fixators can be designed with a lower profile if this region is utilised and patient tolerance improved. This study was designed to explore the anatomical constraints of fixation in the peri-acetabular region and ways in which the fixation could be improved.

Examination of both the osteology of the bony pelvis and multiplanar images obtained from CT and MRI scans of the peri-acetabular region indicated that it should be possible to safely insert three pins into this zone with a crossing angle approaching $70^{\circ}$ utilising the anatomical approaches that had been reported in the literature, i.e. anterolateral and direct lateral.

The anterolateral approach to the peri-acetabular region lies between the iliac spines. For many years trauma surgeons $[10,11]$ have utilised this position recognising that they could achieve good bony purchase and stability in managing difficult-to-control pelvic fractures. This anterolateral site has been shown to be biomechanically as, if not more, stable as traditional constructs in controlling such pelvic fractures but with a decided advantage of not obstructing access to the abdomen and simplifying the fixator design [11]. The anatomical risks of anterolateral pin insertion has been investigated by a number of authors who have shown that with a blunt surgical technique and fluoroscopic control there was little risk to the lateral cutaneous nerve of the thigh and that the femoral neurovascular structures are well medial $[11,12]$. The direct lateral periacetabular approach has been employed for arthrodiastasis of the hip but without any reported anatomical or biomechanical studies. Problems and complications utilising this site have been recognised and include muscle transfixion, vascular injury, pin breakage and pin loosening $[3,4,6,14]$. Issues with the longevity of fixation are significant in situations where maintenance of distraction is the key element of treatment. Aldegheri [3] indicated that fixator removal at 6-10 weeks was usually precipitated by the loosening of the pelvic half pins in his series of 80 patients. The use of hydroxyapatite-coated pins and/or extending the zone of fixation onto the anterior ileum appears to have improved pin longevity but not much more than 4 months' duration.

The use of a virtual cadaver allowed the investigators to perform virtual surgery utilising the abovementioned pin positions and determine which configuration would potentially be achievable maximising bony purchase and avoiding neurovascular injury. It was determined that a freehand insertion of the anterolateral pin $15 \mathrm{~mm}$ above the acetabulum as a reference for the other two pins offered the safest approach. The pre-capital pin would lie just anterior to the apex of the hip joint above the anterolateral pin whilst the post-capital pin would lie behind the apex of the hip joint below the anterolateral pin.
The anatomical dissections confirmed that the lateral cutaneous nerve of the thigh is an unavoidable anatomical hazard for a pin inserted into the anterior inferior iliac spine. Grothaus et al. [15] demonstrated that the nerve emerges medial to the ASIS $(6-73 \mathrm{~mm})$ as multiple branches in $27 \%$ of cases under cover of the inguinal ligament and courses laterally. These variations in its location means that it may pass close to the anterior inferior iliac spine and that damage can only be avoided by blunt dissection and the use of drill and soft tissue sleeves during pin insertion $[16,17]$. The femoral neurovascular bundle lies well medial and is at little risk during pin insertion but if the track of the pin is not carefully followed on the internal oblique view by fluoroscopy, then there is risk of penetrating the sciatic notch and potentially injuring the superior gluteal neurovascular bundle and lumbosacral trunk. This study showed that there is at least $10 \mathrm{~cm}$ of bone available for pin purchase in the adult pelvis that correlates well with the biomechanical studies of pin fixation at this site [10, 12]. Noordeen et al. [12] investigated the mode of failure of pins inserted into either anterosuperior (behind the ASIS) or anteroinferior (above the AIIS) sites in response to a lateral closing force. The anteroinferior site failed gradually and at a higher load than the anterosuperior site indicating that within the confines of the bony pelvis this peri-acetabular site was suitable for external fixation.

Penetration of the hip joint is a potential complication in peri-acetabular external fixation. Haidukewych et al. [11] considered capsular penetration to be synonymous with joint penetration and therefore proposed that the anterolateral pin should be inserted at least $20 \mathrm{~mm}$ above the hip to avoid incursion. As the fibrous hip capsule reflects superiorly onto the outer table of the pelvis (peri-acetabular region) it is firmly attached to bone and for the most part devoid of a synovial lining. Transgression of the capsule in this zone is clearly extra-articular and pin site infection is unlikely to lead to direct joint involvement. For this reason it was felt that the fixation could be brought closer to the joint where the bony cross-sectional area is larger to maximise the extent of bony fixation by all pins.

Transfixation of the gluteus medius and minimus is inevitable during insertion of laterally placed pins and the superior gluteal neurovascular bundle is at risk. The superior gluteal nerve divides into branches soon after passing over the piriformis muscle. The usual pattern is into superior and inferior branches (IB) but in a significant number of cases there is a separate most inferior branch (MIB). In a study of 20 cadavers, Bos et al. [18], found that the distance from the tip of the greater trochanter to the IB or MIB of the superior gluteal nerve ranged from 33 to $63 \mathrm{~mm}$. These inferior branches are the main nerve supply to gluteus medius and tensor fascia lata and at particular 
risk during a lateral approach to the hip joint when the gluteal muscles are split. Insertion of pins in the peri-acetabular region is a more limited procedure but still puts these nerves at risk. In this study the majority of the precapital pins passed above the MIB whilst the reverse was true for the post-capital pin. In our dissections the more common spray pattern of nerve distribution was evident, which to some extent mitigates complete nerve injury as could occur if a neural trunk pattern, were present [19].

The deep inferior branch of the superior gluteal artery passes with the IB or MIB of the superior gluteal nerve and is also at risk of injury. Canadell et al. [4] reported that one of his patients in a series of nine hip distractions required a second operation to control bleeding from a "collateral branch of the superior gluteal artery" indicating that damage to this vessel is a possibility.

The proximity of the post-capital pin to the sciatic notch and the accidental slippage of one pin into this site place the sciatic nerve at risk. This pin traverses a narrower width of peri-acetabular bone compared to the pre-capital pin ( 26 vs. $32 \mathrm{~mm}$ ), is closer to the hip joint margin ( 9 vs. $22 \mathrm{~mm}$ ) and penetrates the internal pelvis under very little muscle cover (iliopsoas). As significant care needs to be taken when inserting this pin to avoid serious complication, an alternative is to insert this pin posterolaterally at an angle of $30^{\circ}$ to the coronal plane. This position places the pin more at right angles to the peri-acetabular surface decreasing the risk of slippage and simultaneously directs the pin away from the sciatic notch, moves the insertion point further posterior to the acetabular margin, increases the depth of bone penetration and makes the pin more likely to exit under cover of muscle (Fig. 4).

The principles of biomechanical stability of external fixation would indicate that pin diameter, number and spread combined with pin clamp and clamp-bar interfaces need to be addressed individually and together in order to achieve a maximally performing construct in the clinical setting. In the standard cantilever-loaded mode, the monolateral fixator is at risk of pin loosening and breakage. During failure the distractive force is lost and the desired biological effect reduced. Modifications of this frame have been developed to counter this problem by which fixation is supplemented by additional lateral pins or by extending the fixation to the iliac crest [14]. In this study we have been able to show that it is possible to introduce three $6-\mathrm{mm}$ pins into the peri-acetabular region with an angular separation of $55^{\circ}$. Studies by Johnson and Fischer [20] showed that as angular separation increases from $0^{\circ}$ to $90^{\circ}$, the bending stiffness also increases. The increased insertion angle of the post capital pin reduces the risk of sciatic nerve damage and improves the stability of fixation by increasing the angular separation between pins.

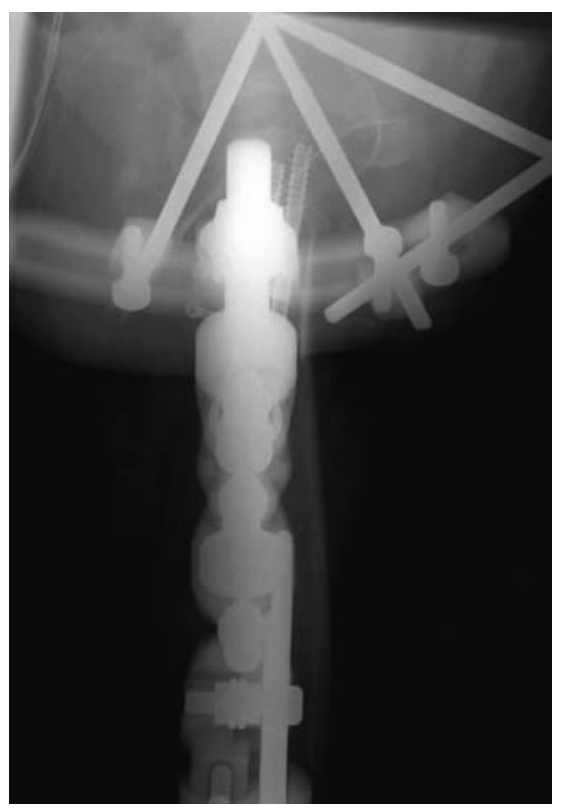

Fig. 4 Lateral radiograph of the modified pin positions in the periacetabular region

This study has demonstrated that it is possible to introduce three pins into the peri-acetabular region of the hip and also that the lateral femoral cutaneous, inferior branch of the superior gluteal and the sciatic nerve are at risk unless a careful technique is employed. It is our recommendation that an image intensifier guided cannulated drill technique is used to insert the anterolateral pin initially and that this be used as a stable platform onto which two lateral pins are introduced. The pre-capital pin should be in the coronal plane above the anterolateral pin and the postcapital pin should be inclined forward by $30^{\circ}$ below the anterolateral pin (Figs. 5, 6). The introduction of a stable anterolateral pin has the ability to enhance the

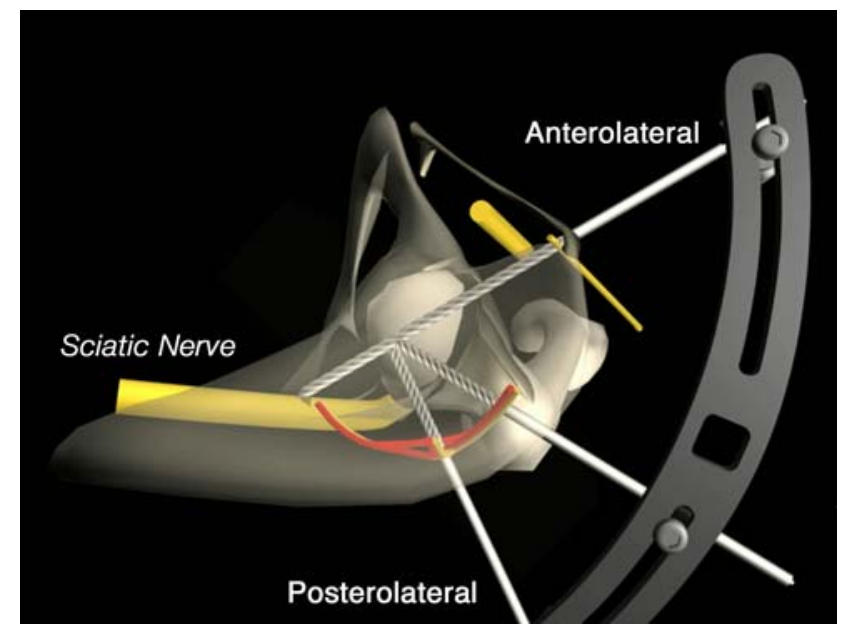

Fig. 5 Modified pin positions transverse plane 


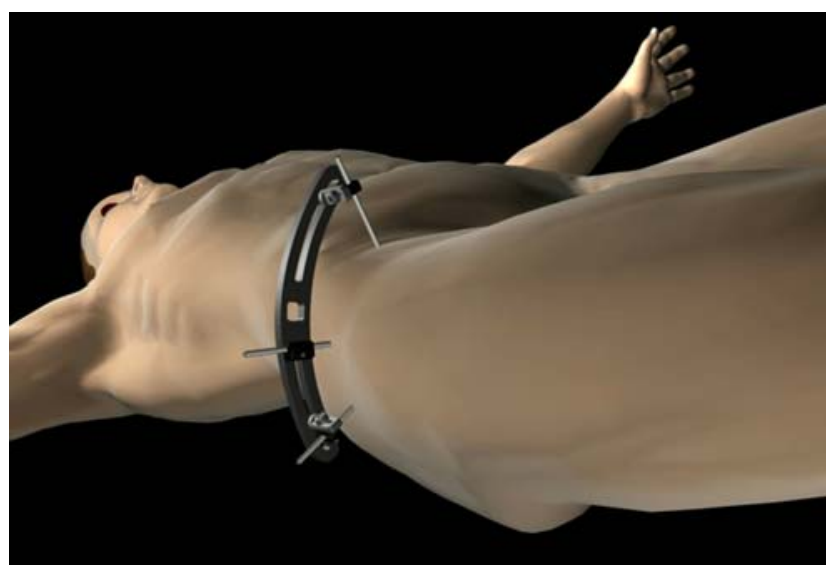

Fig. 6 Modified pin positions caudal view

biomechanics of this form of fixation potentially providing a more stable platform on which to base the transarticular fixation of the hip joint.

\section{References}

1. Routt M, Nork S, Mills W (2000) Percutaneous fixation of pelvic ring disruptions. Clin Orthop 375:15-29

2. Scher D, Jeong G, Grant A, Lehman W, Feldman D (2001) Hip Arthodesis in adolescents using external fixation. J Pediatr Orthop 21:194-197

3. Aldegheri R, Trivella G, Saleh M (1994) Articulated distraction of the hip. conservative surgery for arthritis in young patients. Clin Orthop 301:94-101

4. Canadell J, Gonzales F, Barrios R, Amillo S (1993) Arthrodiastasis for stiff hips in young patients. Int Orthop 17:254-258

5. Kucukkaya M, Kabukcuoglu Y, Ozturk I, Kuzgun U (2000) Avascular necrosis of the femoral head in childhood: the results of treament with articulated distraction method. J Pediatr Orthop 20:722-728

6. Maxwell S, Lappin K, Kealey W, McDowell B, Cosgrove A (2003) Arthrodiastasis in Perthes' disease. J Bone Joint Surg Br $86: 244-250$
7. Kocaoglu M, Kilicoglu O, Goksan S, Cakmak M (1999) Ilizarov Fixator for the treatment of Legg-Calve-Perthes disease. J Pediatr Orthop 8:276-281

8. Salter R, Simmons D, Malcolm B, Rumble E, MacMichael D, Clements N (1980) The biological effect of continuous passive motion on the healing of full thickness defects in articular cartilage. J Bone Joint Surg Am 62:1232-1251

9. Aldegheri R, Trivella G, Saleh M (2000) Articulated distraction of the hip. In: de Bastiani G, Apley G, Goldberg A (eds) Orthofix external fixation in trauma and orthopaedics. Springer, London, pp 605-612

10. Kim W, Hearn T, Seleen O, Mahalingam E, Stephen D, Tile M (1999) Effect of pin location on stability of pelvic external fixation. Clin Orthop 361:237-44

11. Haidukewych G, Kumar S, Prpa B (2003) Placement of half-pins for supra-acetabular external fixation: an anatomic study. Clin Orthop 411:269-273

12. Noordeen M, Taylor B, Briggs T, Lavy C (1993) Pin placement in pelvic external fixation. Injury 24:581-584

13. Ilizarov G (1992) In: Ilizarov GA (ed) Transosseous oseosythnesis. Springer, New York

14. Thacker M, Feldman.D, Madan S, Straight J, Scher D (2005) Hinged distraction of the adolescent arthritic hip. J Pediatr Orthop 25:178-182

15. Grothaus M, Holt M, Mekhail A, Ebraheim N, Yeasting R (2005) Lateral femoral cutaneous nerve: an anatomic study. Clin Orthop 437:164-168

16. de Ridder V, de Lange.L, Popta J (1999) Anatomical variations of the lateral femoral cutaneous nerve and the consequences for surgery. J Orthop Trauma 13:207-211

17. Hospodar P, Ashman E, Traub J (1999) Anatomic study of the lateral femoral cutaneous nerve with respect to the ilioinginal surgical dissection. J Orthop Trauma 13:17-19

18. Bos J, Stoeckart R, Klooswijk A, van Linge B, Bahadoer R (1994) The surgical anatomy of the superior gluteal nerve and anatomical radiologic basis of the direct lateral approach to the hip. Surg Radiol Anat 16:253-258

19. Jacobs L, Buxton R (1989) The course of the superior gluteal nerve in the lateral approach to the hip. J Bone Joint Surg Am 71:1239-1243

20. Johnson W, Fischer D (1983) Skeletal stabilization with a multiplane external fixation device. Biomechanical evaluation and finite element model. Clin Orthop 180:34-43 